Посвяшается памяти A.E. Зотикова

'ФГБНУ «Научноисследовательский институт ревматологии им. В.А. Насоновой» 115522, Российская Федерация, Москва, Каширское шоссе, 34a 2ФГБУ «Национальный медицинский исследовательский центр хирургии имени А.В. Вишневского» Минздрава России 117997, Российская Федерация, Москва, ул. Большая Серпуховская, 27

'V.A. Nasonova Research Institute of Rheumatology 115522, Russian Federation, Moscow, Kashirskoye Highway, 34A

${ }^{2}$ A.V. Vishnevsky National Medical Research Center of Surgery 117997, Russian Federation, Moscow, Bolshaya

Serpukhovskaya str., 27

Контакты: Бекетова Татьяна Валентиновна, tvbek22@ rambler.ru Contact: Tatiana Beketova, tvbek22@rambler.ru

Поступила 30.04.2021

Принята 21.12.2021

Современные возможности визуализации сосудистого повреждения при системных васкулитах крупных сосудов

\author{
Т.В. Бекетова', В.А. Зеленов', А.Е. Зотиков²
}

Инструментальные методы визуализации сосудистого повреждения имеют ключевое значение для диагностики системных васкулитов (СВ) крупных сосудов, оценки эффективности лечения и раннего выявления рецидивов. Несмотря на стремительное развитие ревматологии с углублением представлений о патогенезе $\mathrm{CB}$, внедрением инновационных стратегий лечения и расширением методов диагностики, в настоящее время не существует универсального метода визуализации, который мог бы предоставить исчерпывающую информацию о состоянии крупных сосудов. Стандартизация инструментальных методов для оценки степени активности и диагностика рецидива относятся к ключевым нерешенным проблемам ведения пациентов с СВ крупных сосудов. В 2018 году были опубликованы рекомендации Европейской антиревматической лиги (EULAR, European League Against Rheumatism), осветившие широкий круг проблем визуализации поражения крупных сосудов при CB, которые стали результатом систематизированного анализа современных научных достижений и клинического опыта. Целью данной публикации является обобщение современных возможностей инструментального обследования больных системными васкулитами крупных сосудов с общей характеристикой основных положений рекомендаций EULAR и обсуждением некоторых дискуссионных вопросов.

Ключевые слова: системный васкулит крупных сосудов, гигантоклеточный артериит, артериит Такаясу, аортит, ультразвуковое дуплексное сканирование, позитронная-эимссионная томография, компьютерная томография, ангиография, магниитно-резонансная томография, методы диагностики Для цитирования: Бекетова ТВ, Зеленов ВА, Зотиков АЕ. Современные возможности визуализации сосудистого повреждения при системных васкулитах крупных сосудов. Научно-практическая ревматология. 2022;60(1):91-101

\title{
CURRENT IMAGING TECHNIQUES IN LARGE VESSEL VASCULITIS
}

\author{
Tatiana V. Beketova ${ }^{1}$, Vasily A. Zelenov ${ }^{1}$, Andrey E. Zotikov ${ }^{2}$
}

Instrumental imaging modalities play an important role in the diagnosis, evaluation of treatment efficacy and early detection of recurrences in patients with large vessel systemic vasculitis (SV). There's no currently available universal imaging modality yielding comprehensive information about large vessels regardless all spectacular progress in rheumatology with associated significant insights into SV pathogenesis, innovative therapeutic strategies and broadened diagnostic approaches. Therefore, standardization of instrumental methods in order to accurately assess the disease activity and recognize timely its' relapse remains a pending issue in management of patients with large vessel SV.

Published in 2018 the EULAR (European League Against Rheumatism) guidelines highlighted a wide range of problems concerning visualization of large vessel lesions in SV, resultant from a systematic analysis of up-to-date scientific achievements and clinical experience. The intent of this publication is to summarize the potential of available imaging modalities for patients with large vessel SV in the context of main provisions in the EULAR guidelines and discuss some controversial issues.

Key words: large vessel vasculitis, giant cell arteritis, Takayasu arteritis, aortitis, doppler ultrasonography, positron emission tomography, computed tomography, angiography, magnetic resonance imaging, imaging in diagnosis For citation: Beketova TV, Zelenov VA, Zotikov AE. Current imaging techniques in large vessel vasculitis. NauchcnoPrakticheskaya Revmatologia = Rheumatology Science and Practice. 2022;60(1):91-101 (In Russ.). doi: 10.47360/1995-4484-2022-91-101

Стремительное развитие ревматологии способствовало углублению представлений о патогенетических механизмах системных васкулитов (СВ) с разработкой и внедрением инновационных стратегий с применением генно-инженерных биологических препаратов и расширением методов диагностики. Инструментальные методы визуализации сосудистого повреждения имеют ключевое значение как для диагностики СВ крупных сосудов, так и для оценки эффективности лечения, раннего выявления рецидивов заболеваний.

В соответствии с современной классификацией СВ группа заболеваний с преимущественным поражением крупных сосудов включает артериит Такаясу (АТ) и гигантоклеточный артериит (ГКА) [1]. Кроме того, поражение крупных сосудов, в первую очередь аортит, может сопровождать течение заболеваний из группы вариабельных СВ, таких как болезнь Бехчета и болезнь Когана, выявляться в отдельных случаях ассоциированных с выделением антинейтрофильных цитоплазматических антител (АНЦА-ассоциированных) $\mathrm{CB}$, у пациентов со спондилоартритом, хроническим воспалительным заболеванием кишечника, IgG4-связанным заболеванием, рецидивирующим полихондритом, редко при системной красной волчанке, ревматоидном артрите, и др. [1-6].

Для АТ характерно множественное сегментарное воспалительное поражение аорты 
и ее крупных ветвей с развитием стенозов, окклюзий, редко - с формированием аневризм. У ряда пациентов с АТ обнаруживают вовлечение в воспалительный процесс коронарных (10-45\%) и легочных артерий [7, 8], что учтено при разработке диагностических критериев АТ [9]. Ключевое значение в определении варианта течения АТ представляет подробное инструментальное обследование с выявлением локализации поражения аорты и ее ветвей, в соответствии с которой предложена ангиографическая классификация, подразделяющая АТ на пять типов [10] (рис. 1) с дополнительным выделением поражения коронарных и легочных артерий (рис. 2).

В то время, как в азиатских странах АТ наиболее часто характеризуется поражением дуги аорты [11], в российской популяции преобладает V тип АТ с генерализованным поражением (57\%) [12], который, по данным многовариантного анализа [13], ассоциируется с наибольшим риском рецидива. По данным российской когорты, у пациентов с АТ может развиваться обширное поражение брахиоцефальных артерий (рис. 3) вплоть до окклюзии всех ветвей дуги аорты, получившее название «синдром лысой дуги». Заинтересованность легочной артерии встречается редко, тем не менее иногда выявляют сужение основных стволов легочной артерии. При поражении торакоабдоминального отдела аорты чаще отмечают множественную патологию всех висцеральных и почечных артерий (рис. 4) с развитием тяжелой артериальной гипертензии, которая закономерно существенно ухудшает прогноз. На поздней стадии АТ у молодых пациентов формируется тотальный кальциноз грудной аорты (рис. 5). Другим патогномоничным для АТ признаком является расширение и удлинение дуги Риолана при поражении чревного ствола и верхней брызжеечной артерии. Следует отметить, что ни при одном из других

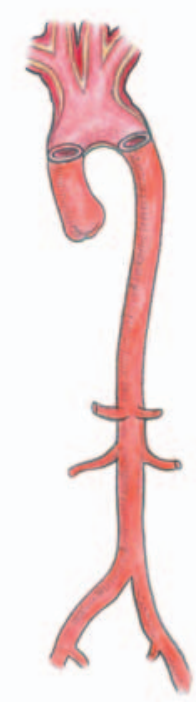

a

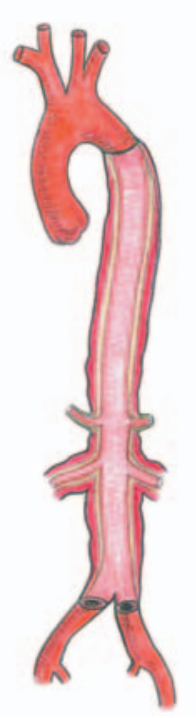

$\Gamma$

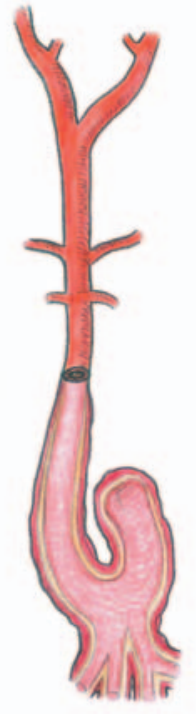

6

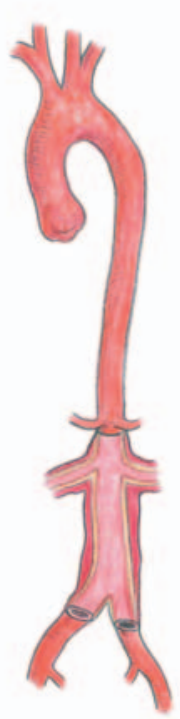

Д

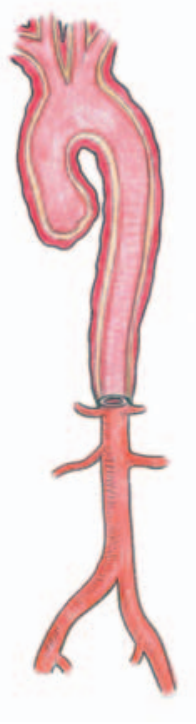

B

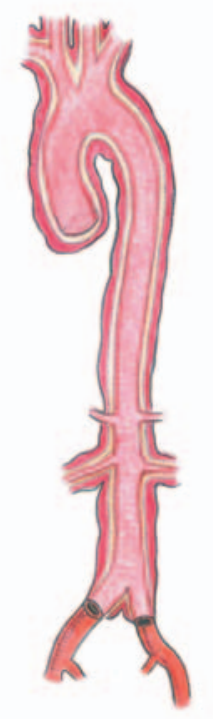

e

Рис. 1. Классификация артериита Такаясу: а - I тип, поражение ветвей дуги аорты; б - ІІа тип, поражение восходящей аорты, дуги и ее ветвей; в - IIb тип, поражение восходящей аорты, дуги и ее ветвей, а также нисходящей грудной аорты; г - III тип, поражение нисходящей грудной, брюшной аорты и ее ветвей; $\boldsymbol{\boldsymbol { A }}$ - IV тип, поражение брюшной аорты и ее ветвей (без грудной аорты); $\boldsymbol{e}-V$ тип, вся аорта и ее ветви 


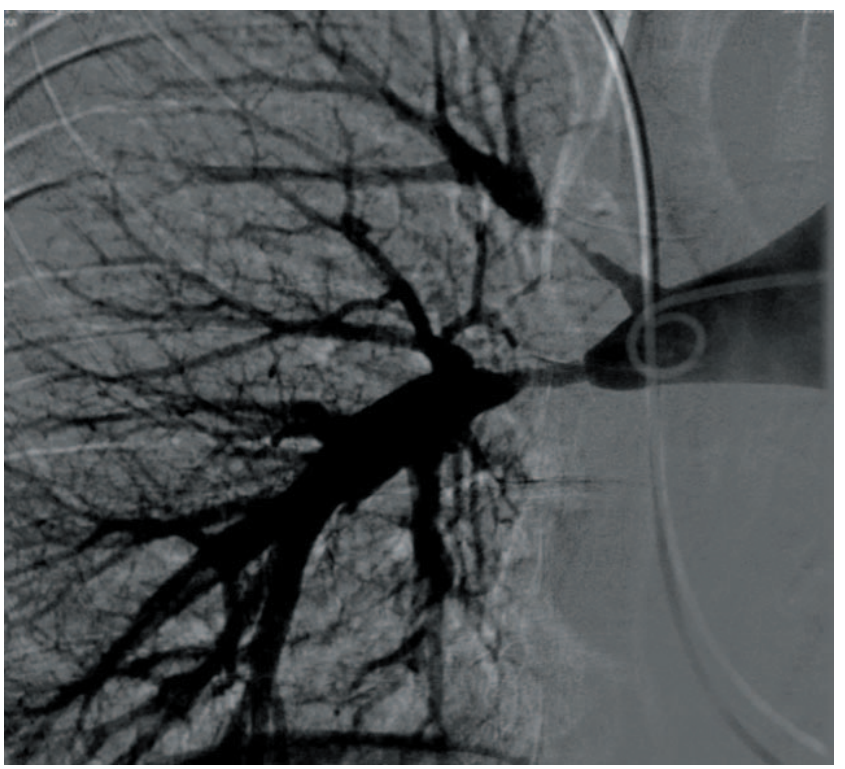

Рис. 2. Селективная ангиопульмонография у пациентки с артериитом Такаясу и поражением легочных артерий: субтотальный стеноз в устьях и проксимальных сегментах верхнедолевой и нижнедолевой ветвей (стрелки) правой легочной артерии

заболеваний коллатерали не бывают столь мощно развиты, как при АТ.

При ГКА, наиболее распространенной форме СВ крупных сосудов, наблюдается поражение аорты и/или ее главных ветвей, преимущественно брахиоцефальных, наиболее часто - височной артерии. Примерно у трети больных ГКА поражение артерий, кровоснабжающих глаза, приводит к развитию ишемической оптической нейропатии и слепоте, что может быть первым проявлением заболевания. Значительно реже выявляют поражение аорты (преимущественно в грудном отделе с развитием аневризм, в том числе расслаивающих) и ее ветвей (сонных, подключичных, позвоночных, коронарных артерий), интракраниальных артерий. К факторам риска развития дилатации аорты у пациентов с ГКА относят поражение аорты в активную фазу болезни, артериальную гипертензию, мужской пол и курение $[14,15]$. Визуализация височных артерий при помощи ультразвукового дуплексного сканирования (УЗДС) или МРТ-ангиографии позволяет выявить типичные изменения в 77-73\% случаев ГКА, при этом данные неинвазивные методы визуализации имеют примерно одинаковую диагностическую ценность в сравнении с биопсией височной артерии [16, 17]. Выявление при УЗДС височных артерий феномена «ореола» («halo»; рис. 6) демонстрирует высокие чувствительность и специфичность (77\% и 96\% соответственно) [18]; другим патогномоничным для ГКА признаком является стойкость гипоэхогенной зоны, несмотря на сжатие просвета артерии ультразвуковым зондом, с чувствительностью 77-79\% и специфичностью $100 \%[19,20]$.

Несмотря на расширение возможностей инструментального обследования, в настоящее время не существует универсального способа визуализации, который мог бы предоставить исчерпывающую информацию о состоянии сосудов; отдельные методы имеют определенное и взаимодополняющее значение (табл. 1). Стандартизация инструментальных методов для оценки степени активности и диагностика рецидива относятся к ключевым нерешенным

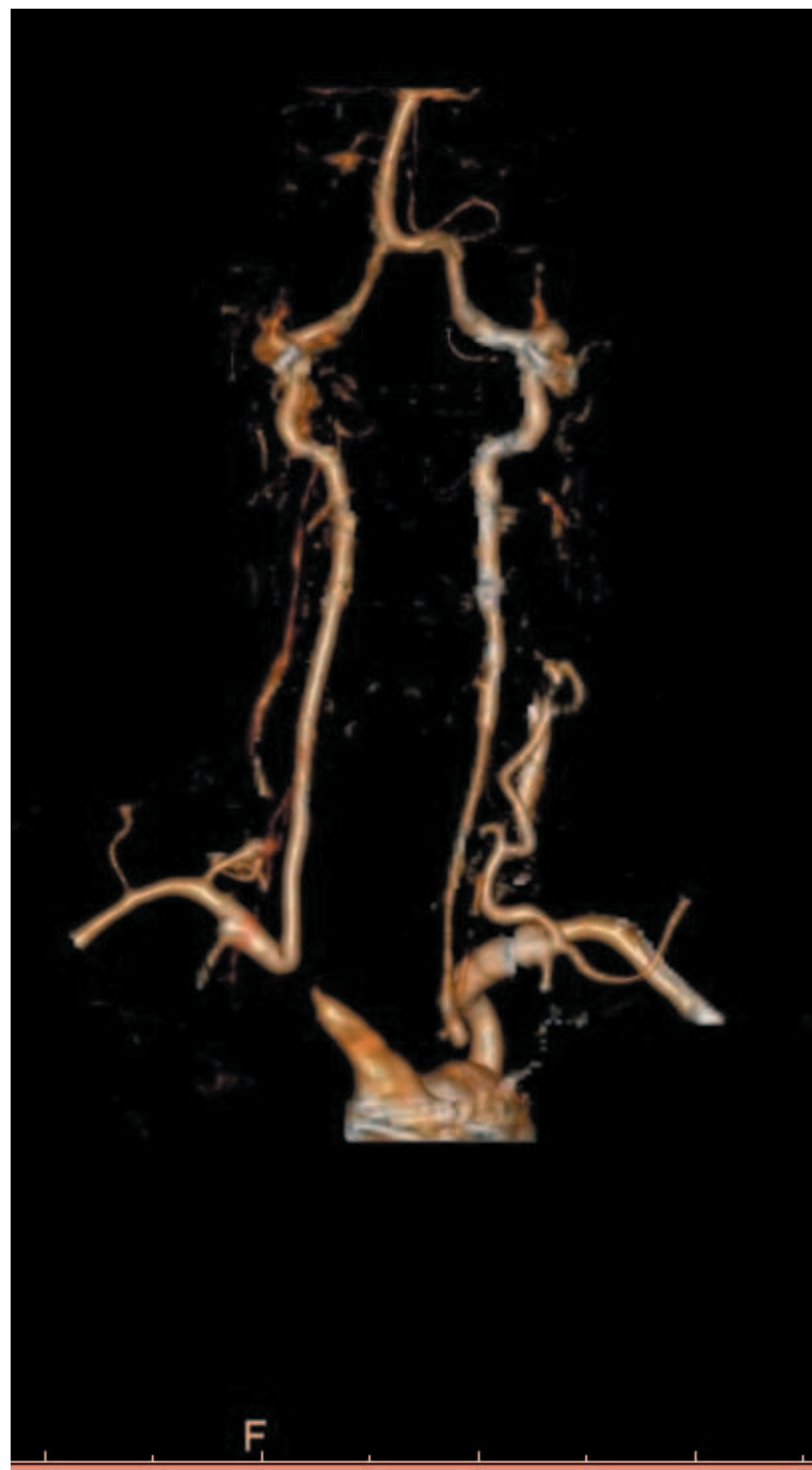

Рис. 3. Трехмерная реконструкция КТ-ангиографии у пациентки c AT: окклюзия брахиоцефального ствола, обеих общих сонных артерий, синдром позвоночно-подключичного обкрадывания справа (кровоснабжение головного мозга за счет левой позвоночной артерии)

проблемам ведения пациентов с СВ крупных сосудов. Хорошо известно, что у пациентов с несомненной клинической и лабораторной ремиссией АТ при гистологическом исследовании в стенке артерий могут обнаруживаться признаки активного васкулита [21, 22]. Морфологическая ремиссия АТ наступает не ранее, чем через 3-4 месяца после достижения лабораторной ремиссии, что учитывают при определении сроков оперативного лечения [12]. G. Kerr и соавт. [22] у 44\% больных с отсутствием клинических признаков активности АТ при гистологическом исследовании обнаруживали активное воспаление в стенке артерий. T. Xenitidis и соавт. [23] на фоне лечения ингибитором интерлейкина 6 тоцилизумабом (ТЦЗ) отметили прогрессирование поражения стенки аорты при АТ, несмотря на стойкую нормализацию уровня С-реактивного белка (СРБ). По данным D. Spira и соавт. [24], несмотря на нормализацию уровней СРБ, скорости оседания эритроцитов 


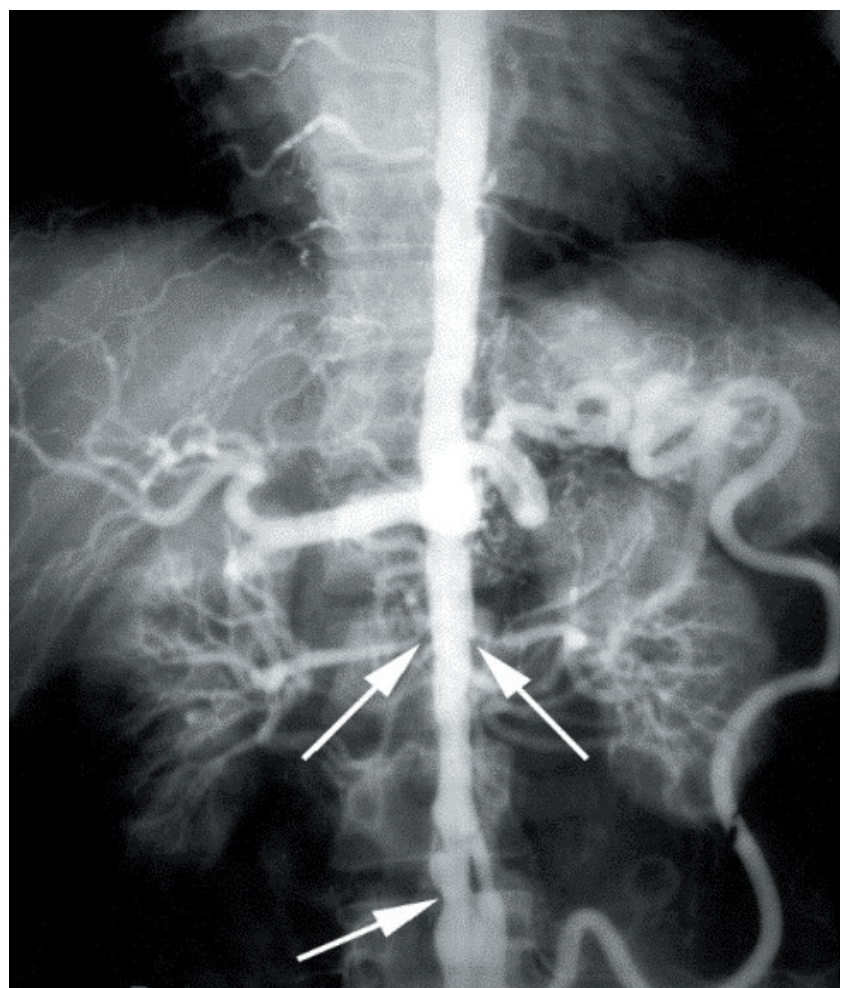

Рис. 4. Рентген-контрастная ангиография у пациентки с АT: стенозы супра-, интер- и инсраренального отделов аорты (нижняя стрелка), верхней брыжеечной артерии, обеих почечных артерий (верхние стрелки)

и исчезновение клинических признаков активности АТ на фоне лечения ТЦЗ, в одном из двух случаев результаты МРТ свидетельствовали о персистенции воспаления в сосудах. При ГКА развитие аневризмы аорты возможно при отсутствии клинических признаков активности и может произойти спустя годы после начала заболевания [25].

Значение инструментальных исследований для установления диагноза СВ с поражением крупных сосудов, уточнения локализации поражений и мониторинга активности трудно переоценить, однако эти методы не всегда надежны [16, 26-28] и имеют ряд недостатков (табл. 2).

Информативность инвазивной рентгеноконтрастной ангиографии, которая в течение десятилетий оставалась «золотым стандартом» для диагностики АТ и других СВ крупных сосудов, имеет ограничения, поскольку выявляет изменения просвета сосудов (стеноз, окклюзия, аневризма), но не позволяет оценить состояние сосудистой стенки и может не выявлять небольшие неокклюзивные поражения. Кроме того, выполнение рентген-контрастной ангиографии сопряжено с риском аллергических реакций, ятрогенной эмболизации, формирования гематомы, диссекции артерий.

Тем не менее рентген-контрастная ангиография, как и КТ-ангиография, позволяет выявить патогномоничные рентгенологические симптомы. Так, в случае поражения брахиоцефальных артерий для АТ типична локализация патологического процесса во II и III сегментах подключичных артерий и в общих сонных артериях, в то время как при атеросклерозе изменения, как правило, локализуются в области бифуркации сонных артерий, общие сонные и позвоночные артерии поражаются чаще

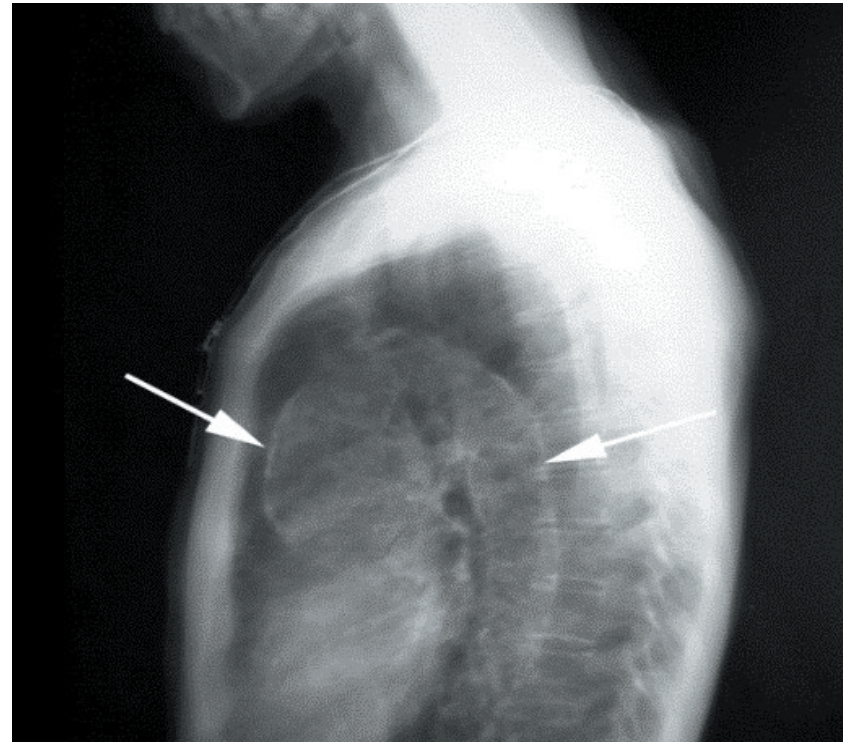

a

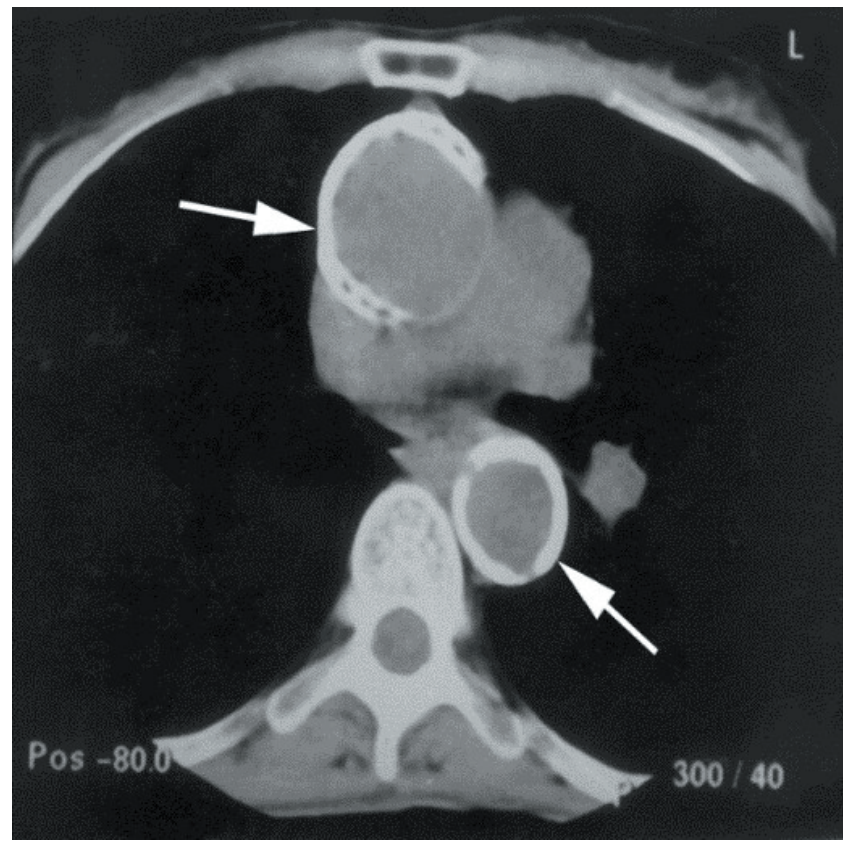

6

Pис. 5. a - рентгенограмма (боковая проекция) пациента с AT и тотальным кальцинозом грудной аорты (стрелки); б - КТ пациента с AT: кальциноз восходящего и нисходящего отделов грудной аорты (стрелки)

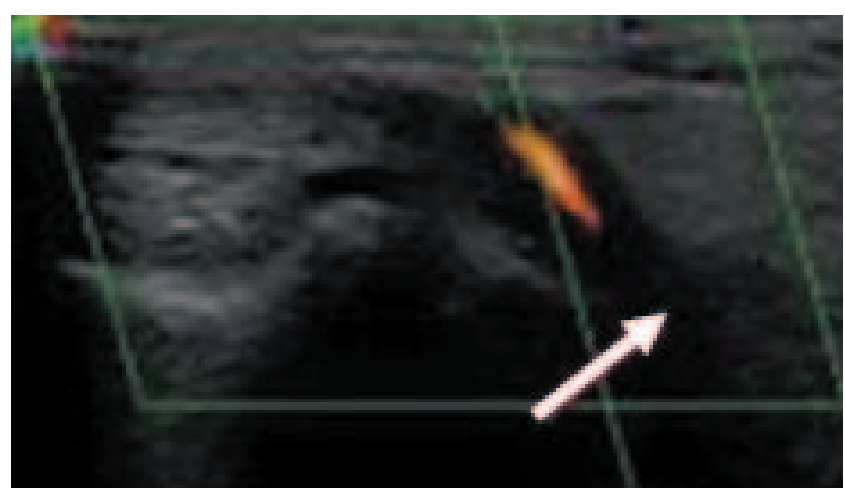

Рис. 6. УЗДС височной артерии у пациентки с ГКА: гипоэхогенный феномен «halo» (стрелка)

Научно-практическая ревматология. 2022;60(1):91-101 
Таблица 1. Сравнительная характеристика основных инструментальных методов, применяемых для визуализации поражения крупных сосудов при СB

\begin{tabular}{|c|c|c|c|c|c|}
\hline Методы & $\begin{array}{l}\text { Характерные для СВ результаты. } \\
\text { Чувствительность } \\
\text { и специфичность при СВ }\end{array}$ & $\begin{array}{l}\text { Оценка структурных } \\
\text { изменений }\end{array}$ & $\begin{array}{l}\text { Оценка } \\
\text { функциональных } \\
\text { изменений }\end{array}$ & $\begin{array}{l}\text { Введение } \\
\text { контрастно- } \\
\text { го вещества }\end{array}$ & Недостатки метода \\
\hline узДС & $\begin{array}{l}\text { ГКА: гипоэхогенный, несжимае- } \\
\text { мый «ореол»; фракультативные } \\
\text { изменения скоростных и потоко- } \\
\text { вых показателей. } \\
\text { AТ: резкое утолщение стенок сон- } \\
\text { ных артерий, «симптом макаро- } \\
\text { на» («Масаroni sign») - расплыв- } \\
\text { чатость контуров. } \\
\text { ГКА [33, 51]: чувствительность } \\
67-77 \%, \text { специфичность 91-96\%. }\end{array}$ & $\begin{array}{l}\text { Неинвазивный способ } \\
\text { определения геометрии } \\
\text { сосуда, протяженности } \\
\text { процесса, осложнений. } \\
\text { Удобно для динамическо- } \\
\text { го наблюдения. }\end{array}$ & $\begin{array}{l}\text { С высоким разре- } \\
\text { шением позволяет } \\
\text { определить функ- } \\
\text { циональные изме- } \\
\text { нения кровотока. } \\
\text { Удобно для дина- } \\
\text { мического наблю- } \\
\text { дения. }\end{array}$ & Не требует & $\begin{array}{l}\text { Анатомические ограничения (невоз- } \\
\text { можность визуализации грудной } \\
\text { аорты). } \\
\text { Малая информативность в отноше- } \\
\text { нии наличия воспалительной актив- } \\
\text { ности. } \\
\text { Зависимость результата от квалифи- } \\
\text { кации оператора }\end{array}$ \\
\hline
\end{tabular}

Относительно низкая чувствительHOCTь.

Сосудистый стеноз, окклюзия, эктазия.

Рентгенконтрастная ангиография

Сохраняет значение как «золотой стандарт» визуализации сосудистого повреждения.

Высокая чувствительность при изменениях в мелких сосудах.
Позволяет определить геометрию сосуда, длину протяженности процесса, осложнения.

Применяют при эндоваскулярном вмешательстве (стентирование).
Не позволяет опре- Требует делить функцио- введения нальные измене- контрастнония кровотока го вещества

Не позволяет оценить состояние сосудистой стенки (наличие воспалительной активности) и окружающих тканей, может не выявлять небольшие неокклюзивные поражения.

Ограниченная доступность, высокая стоимость.

Зависимость результата от квалификации оператора.

Значительная лучевая нагрузка.

Потенциальный риск аллергических реакций, эмболизации, гематомы, диссекции артерий

Не дает информации об изменениях

C высоким разрешением и широким диапазоном сканирования позволяет определить структурные изменения в сосудах (просвет, стенки), оценить протяженность процесса, осложнения
Не позволяет опре- Использоделить функцио- вание йоднальные измене- содержащения кровотока го контраста в сосудистой стенке (наличие воспалительной активности).

Потенциальная нефротоксичность контрастного вещества.

Значительная лучевая нагрузка (15 м3в).

C высоким разрешением

Утолщение и уплотнение сосудистой стенки; стенозы магистраль- и широким диапазоном ных артерий, окклюзия, эктазия. позволяет определить

Отек стенки сосуда, реакция окру- структурные изменения жающей ткани.

MPTангиография Последствия (инфаркт, некроз, кровотечение).

ГКА [51]: чувствительность 69\%, специфичность $91 \%$.

ГКА [18]: чувствительность 73\%, специфичность $88 \%$.

АТ $[49,50]:$ чувствительность $100 \%$, специфичность 100\%.

Утолщение сосудистой стенки и поглощение радиофармпрепарата.

Сосудистый стеноз, окклюзия, эктазия.

(протяженность процесса, осложнения), но с меньшей точностью чем КТ-ангиография

Возможна оценка воспалительного процесса.

Доступна любая локализация (легочные, внутричерепные, экстракраниальные и крупные сосуды).

C высоким разрешением и широким диапазоном сканирования позволяет оценить структурные-

пэт (ФДГ)-кТ Отек стенки сосуда, реакция окружающей ткани.

Наиболее раннее выявление рецидива Св.

Экстракраниальный ГКА [39, 40]: чувствительность 67-77\%, специфричность 66-100\%. изменения в сосудах

(просвет, стенки) и окружающих тканях.

Дает информацию о воспалительной активности в сосудистой стенке.
Менее информативен, чем КТ-ангиография.

Не стандартизирован.

Не позволяет определить функциональные изменения кровотока

Не требует Ограниченная доступность, трудоемкость, высокая стоимость.

Техническая вариабельность.

Зависимость результата от квалисрикации оператора.

Потенциальная нефротоксичность и нейротоксичность гадолиния.
Не дает информации об изменениях во внутричерепных сосудах.

Не позволяет опре- Требует вве- Относительно низкая чувствительделить функцио- дения ради- ность.

нальные измене- офарм-пре- Ограниченная доступность, высокая ния кровотока парата стоимость.

Значительная лучевая нагрузка (3,7-13,9 м3в).

Примечание: СВ - системный васкулит; УЗДС - ультразвуковое дуплексное сканирование; ГКА - гигантоклеточный артериит; АТ - артериит Такаясу; КТ - компьютерная томография; МРТ - магнитно-резонансная томография; ПЭТ (ФДГ)-КТ - позитронно-эмиссионная томография/компьютерная томография с введением 18F-фртордезоксиглюкозы 
Таблица 2. Рекомендации EULAR по применению методов визуализации при CB крупных сосудов в клинической практике [16]

\begin{tabular}{lll}
\hline & Уровень & \multicolumn{1}{c}{ Степень согласия экспертов: } \\
Рекомендации & среднее значение (стандарт- \\
& доказательности & ное отклонение);\% экспертов \\
(ОСЕВМ) & оценкой $\geq 8$
\end{tabular}

1. Пациентам с подозрением на ГКА рекомендовано раннее инструментальное обследование в дополнение к клиническим методам диагностики ГКА с учетом доступности метода визуализации и квалификации специалистов. Диагностическое обследование не должно задерживать начало лечения.

2. У пациентов с высокой вероятностью ГКА и положительными результатами одного из инструментальных методов ГКА может быть диагностирован без дополнительного обследования (биопсия или другие методы визуализации). При низкой вероятности ГКА и отрицательных результатах 2 инструментального исследования диагноз ГКА можно считать отвергнутым. Во всех сомнительных случаях требуется дополнительное обследование для установления диагноза.

3. У пациентов с подозрением на ГКА с краниальными симптомами в качестве первого инструментального метода исследования рекомендовано УЗДС височных, подмышечных артерий. При этом наиболее патогномоничным признаком является несжимаемый «ореол» («halo»).

4. МРТ высокого разрешения краниальных артерий может быть использована как альтернативный метод исследования состояния стенки сосудов для диагностики ГКА при неинсоормативности 2 или недоступности УЗДС.

5. КТ и ПЭТ не рекомендованы для оценки воспаления краниальных артерий.

$9,2(2,1)$

$90 \%$ с оценкой $\geq 8$

6. УЗДС, ПЭТ, МРТ и/или КТ могут быть использованы для диагностики ГКА с экстракраниальным поражением для выявления воспаления стенок сосудов и/или изменения просвета экстракраниальных артерий. УЗДС имеет ограниченную ценность для оценки поражения грудной аорты.

7. У пациентов с подозрением на АТ в первую очередь следует использовать МРТ для выявления воспаления стенок сосудов и/или изменения их просвета, что предполагает высокую квалифика- 3 цию специалиста и доступность метода.

8. ПЭТ, КТ и/или УзДС могут быть использованы в качестве альтернативных методов визуализации у пациентов с подозрением на АТ. УЗДС имеет ограниченное значение для оценки поражения грудной аорты.

9. Рентген-контрастная ангиография не рекомендована для диагностики ГКА или АТ, поскольку может быть заменена другими методами визуализации.

$9,4(1,0)$

$90 \%$ с оценкой $\geq 8$

10. У пациентов с СВ крупных сосудов (ГКА или АТ) с подозрением на рецидив необходимо выполнение одного из инструментальных методов обследования для подтверждения или исключения активности Св. Как правило, инструментальные исследования не рекомендованы пациентам с клинической и лабораторной ремиссией.

11. У пациентов с СВ крупных сосудов (ГКА или АТ) МРТ-ангиография, КТ-ангиография и/или УЗДС могут быть использованы для мониторинга структурных повреждений сосудов, в частности для выявления стеноза, окклюзии, дилатации и/или аневризм. Частоту скрининга, а также метод визуализации следует определять индивидуально.

12. Интерпретация исследования должна выполняться хорошо обученным специалистом, владеющим соответствующим оборудованием. Надежность визуализации нередко вызывает сомнения, но может быть повышена благодаря специальной подготовке. Предложения по технике выполнения и эксплуатации различных методов представлены в особом разделе данных рекомендаций.

$9,7(0,6)$

$100 \%$ с оценкой $\geqq 8$

$9,2(1,1)$

$90 \%$ с оценкой $>8$

\section{$9,5(1,2)$}

$95 \%$ с оценкой $>8$

$9,8(0,6)$

$5-$ МРТ и УзДС

$100 \%$ с оценкой $\geqq 8$

3

$9,1(1,4)$

$90 \%$ с оценкой $>8$

$3-$ КТ
$5-$ - ПЭТ и УзДс

3 - КТ
5 - ПЭТ и УзДС

$9,4(0,8)$

$100 \%$ с оценкой $\geqq 8$

5

$9,8(0,6)$

$100 \%$ с оценкой $\geq 8$

$9,4(0,8)$

$100 \%$ с оценкой $\geq 8$

$9,3(1,2)$

$95 \%$ с оценкой $\geq 8$

$9,8(0,6)$

$100 \%$ с оценкой $\geqq 8$

Примечание: EULAR - Европейская антиревматическая лига (European League Against Rheumatism); OCEBM - Оксфордский центр доказательной медицины (Oхford University Centre for Evidence-Based Medicine); ГКА - гигантоклеточный артериит; УЗДС - ультразвуковое дуплексное сканирование; МРТ - магнитно-резонансная томограсрия; КT - компьютерная томография; ПЭТ - позитронно-эмиссионная томографии; $A T$ - артериит Такаясу; $C B$ - системный васкулит

в устье, подключичные артерии - как правило, в I сегменте, что нередко вызывает развитие синдрома позвоночно-подключичного обкрадывания. Вместе с тем при атеросклерозе может развиваться обширное поражение ветвей дуги аорты, имеющее сходство с АТ, при этом атеросклеротический процесс распространяется на наружные и внутренние сонные артерии, вызывая гемодинамически значимые стенозы, иногда - окклюзии. При проксимальном типе атеросклеротического поражения характерны стеноз или окклюзия брахиоцефального ствола. Для дифференциальной диагностики между СВ крупных сосудов и ранним атеросклеротическим поражением важное значение приобретает выполнение УЗДС.

Ультразвуковой метод обладает высоким разрешением и позволяет осуществлять частый мониторинг поражения сосудов доступных локализаций, но исследование грудной аорты требует транспищеводного доступа [27,
29]. Несмотря на исчезновение феномена «halo» у пациентов с ГКА через 2-4 недели лечения глюкокортикоидами [30-35], остаточные изменения в экстракраниальных артериях по данным УЗДС могут сохраняться в течение нескольких месяцев.

Позитронная эмиссионная томография (ПЭТ) проявляет высокую чувствительность при выявлении очагов воспаления сосудистой стенки (рис. 7), в том числе на ранней стадии, когда активация воспалительных клеток может предшествовать появлению структурных изменений [36-40]. ПЭТ-КТ обеспечивает визуализацию сосудистой стенки и изменений в просвете сосуда; кроме того, важным преимуществом ПЭТ является способность идентифицировать другие патологические состояния, такие как инфекции и новообразования. Вместе с тем значение ПЭТ для мониторинга пациентов с СВ крупных сосудов обсуждается. Так, в ряде случаев ГКА, несмотря на ответ 
на лечение, выявляется персистенция поглощения радиофармпрепарата при ПЭТ, что связывают как с остаточным активным воспалением, так и с отражением процесса ремоделирования сосудов. По данным D. Blockmans и соавт. [38], проводивших повторные ПЭТ пациентам с ГКА в дебюте заболевания и на фоне лечения, у 2/3 пациентов с достигнутой полной клинической ремиссией через 3 и 6 месяцев лечения по-прежнему определялось накопление радиофармпрепарата, но его уровень был значительно снижен в сравнении с первоначальным. В двух клинических исследованиях продемонстрированы различные чувствительность (67-77\%) и специфичность (66-100\%) ПЭТ при ГКА, что может быть обусловлено небольшим числом пациентов и использованием разных критериев ГКА $[39,40]$. Кроме того, накопление 18F-фтордезоксиглюкозы в стенке сосудов не является специфичным для васкулита и наблюдается при атеросклеротическом поражении, дифференциация с которым может становиться непростой задачей, особенно в руках неопытного оператора [41, 42].

В последние годы все более широко используют современные методы визуализации, такие как МР-ангиография, КТ-ангиография [18, 26-28, 39, 43-51]. Последний является надежным неинвазивным инструментом визуализации поражения как аорты, так и легочной артерии [45], в том числе на ранней стадии заболевания (до формирования стеноза), используется для ранней диагностики с возможностью мониторинга воспалительной активности СВ [46]. Показано, что для диагностики АТ чувствительность и специфичность КТ-ангиографии достигает 100\% [47], при ГКА - 73\% и 78\% соответственно [39]. КТ-ангиография с трехмерным изображением сосудов позволяет выявить изменения как просвета, так и стенки сосуда исследуемого отдела, но точность измерения толщины и плотности стенки значительно снижается за счет присутствия в сосудистом русле контраста. Кроме того, при КТ-ангиографии используют потенциально нефротоксичные контрастные вещества, и присутствует лучевое воздействие, что существенно ограничивает возможности повторных исследований для мониторинга активности заболевания.

МРТ-ангиография как альтернативный метод без ионизирующего излучения способна сочетать в одном исследовании визуализацию с изображением в трехмерной (3D) плоскости стенок сосудов любой локализации и изучение перфузии органов с целью выявления гипоперфузии, в частности легких [48]. Так, при поражении легочных артерий рентген-контрастная ангиография позволяет выявить изменения в $50 \%$ случаев, в то время как чувствительность и специфичность МРТ-ангиографии достигает $100 \%[49,50]$. При ГКА с поражением поверхностных черепных артерий ретроспективное прямое сравнение МРТ и УЗДС продемонстрировало их сходные чувствительность (69\% и $67 \%$ соответственно) и специфичность $(91 \%$ и $91 \%$ соответственно) [51]. Предполагается, но окончательно не доказано, что усиление контрастом сосудистой стенки может отражать активное воспаление. Ценность МРТ повышает возможность проведения дифференциальной диагностики с другими состояниями. Недостатки МРТангиографии по сравнению с другими методами включают более длительное время сканирования и возможность отдаленных осложнений, таких как нефрогенный системный фиброз и отложение гадолиния в базальных ганглиях головного мозга с неуточненной патогенностью [52, 53]. В связи с этим у пациентов со снижением функции почек

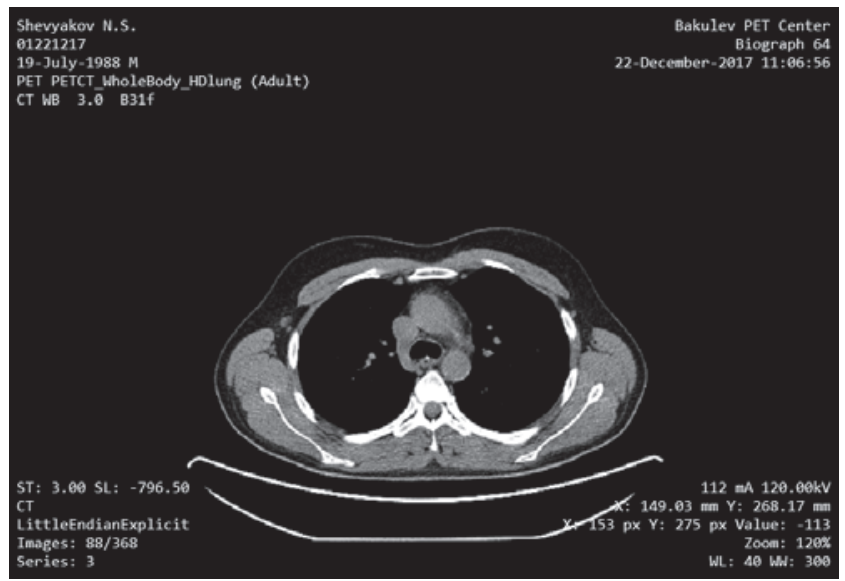

Рис. 7. ПЭТ-КТ: накопление 18F-фртордезоксиглюкозы в стенке дуги аорты (стрелка)

ограничивают применение контрастных препаратов на основе гадолиния (в первую очередь гадодиамида, гадопентетат димеглюмина, гадоверсетамида).

Таким образом, в последние годы прогрессу в диагностике СВ крупных сосудов с расширением возможностей визуализации сопутствовали существенные противоречия, включая неоднозначность выбора приоритетных методов на этапе диагностики и в ходе последующего мониторинга, разногласия в вопросах клинической значимости методов для оценки степени воспалительной активности и повреждения сосудов.

В январе 2018 года были опубликованы рекомендации Европейской антиревматической лиги (EULAR, European League Against Rheumatism) по использованию методов визуализации при СВ крупных сосудов [16], которые аккумулировали современные научные достижения и стали результатом глубокого анализа систематического литературного обзора и мнения 20 экспертов из 10 европейских стран. Рабочая группа экспертов была междисциплинарной и включала наряду с ревматологами - членами EULAR - специалистов лучевой диагностики и ядерной медицины, терапевта, а также представителей среднего медицинского персонала и пациентов. Представленная доказательная база всесторонне обсуждалась, обобщения и формулировки были приняты голосованием участников рабочей группы. Консенсус был принят, если в первом туре за рекомендацию проголосовали более $75 \%$ членов рабочей группы, во втором - более $67 \%$, в третьем - более 50\%. Уровень доказательности положений по данным литературного обзора оценивали в соответствии со шкалой Оксфордского центра доказательной медицины (OCEBM, Oxford University Centre for Evidence-Based Medicine) [54] с градациями от 1 балла (наиболее достоверные доказательства: систематические обзоры поперечных исследований с эталонным стандартом и ослеплением) до 5 баллов (наименее достоверные доказательства: ограничены обоснованием механизма действия).

Результатом систематизированного обобщения современных научных достижений и клинического опыта стали 12 рекомендаций EULAR, осветившие широкий круг проблем применения методов визуализации при CB крупных сосудов в клинической практике (табл. 2).

Рекомендация 1. Пациентам с подозрением на ГКА рекомендовано раннее инструментальное 
обследование в дополнение к клиническим методам диагностики ГКА, при этом выбор метода зависит от клинической картины и диагностических возможностей лечебного учреждения, включая доступность методов визуализации и квалификацию специалистов. Несмотря на то, что при положительных результатах биопсии дополнительные диагностические исследования могут не потребоваться, рабочая группа рекомендует в качестве первой линии диагностики СВ крупных сосудов отдавать предпочтение инструментальным методам визуализации из-за их низкой инвазивности, сопоставимой информативности и возможности оценить широкий спектр локализаций поражения сосудов. УЗДС рассматривается как наиболее эффективный и экономически целесообразный метод диагностики, позволяющий быстро и с высокой вероятностью выявить поражение сосудов и его протяженность. Выполнение диагностического обследования, включая биопсию, не должно препятствовать началу лечения. Исследование следует проводить до начала лечения или в ходе его первой недели, поскольку высокие дозы глюкокортикоидов быстро снижают чувствительность методов визуализации.

Рекомендация 2. Выполнение биопсии рассматривается как наиболее точный метод диагностики ГКА, однако его выполнение в ряде случаев рискованно и/или нецелесообразно, поэтому при высокой вероятности ГКА и положительных результатах одного из инструментальных методов ГКА может быть диагностирован без дополнительного обследования (биопсия или другие методы визуализации). У пациентов с низкой вероятностью ГКА и отрицательными результатами инструментального обследования диагноз ГКА можно считать отвергнутым. Во всех сомнительных случаях необходимо дополнительное обследование для установления диагноза.

Рекомендация 3. У пациентов с подозрением на ГКА с краниальными симптомами (головная боль, расстройства зрения, «хромота» височно-нижнечелюстных суставов, боль и отек височных артерий) приоритетным методом должно быть УЗДС в связи с его высокой информативностью, доступностью, отсутствием лучевой нагрузки и невысокой стоимостью в сравнении с другими методами. Наиболее патогномоничным признаком ГКА является несжимаемый «ореол» («halo»), который определен как «однородное гипоэхогенное утолщение стенки, хорошо очерченное со стороны просвета сосуда, которое определяется как в продольной, так и в поперечной плоскостях, чаще всего концентрическое на поперечном срезе». Поскольку при других СВ (например, при АНЦАассоциированном СВ), инфекционных заболеваниях или выраженном атеросклерозе иногда может обнаруживаться ложноположительный феномен «halo», результаты УЗДС всегда следует интерпретировать с учетом клинических признаков и лабораторных показателей. По мнению экспертов, у пациентов с подозрением на ГКА при отсутствии или неубедительности признаков поражения височных артерий большое значение приобретает выполнение УЗДС подмышечных артерий, которое увеличивает чувствительность на $2 \%$ в сравнении с изолированным УЗДС височных артерий [55].

Рекомендация 4. МРТ высокого разрешения краниальных артерий (височных, затылочных, лицевых) может быть использована как альтернативный метод исследования состояния стенки сосудов для диагностики ГКА при неинформативности или недоступности
УЗДС. Преимуществом МРТ в сравнении с УЗДС является более высокая стандартизация исследования и возможность одновременного исследования краниальных и экстракраниальных артерий, включая аорту, а также интракраниальных артерий, которые в ряде случаев также могут поражаться при ГКА. МРТ краниальных артерий следует выполнять до назначения глюкокортикоидов или в течение первых дней терапии для исключения ложноотрицательных результатов.

Рекомендация 5. Экспертная группа не рекомендует КТ или ПЭТ для оценки воспаления краниальных артерий в связи с отсутствием доказательной базы, лучевой нагрузки, технической сложности и высокой стоимости. Исследования этих методов для оценки краниальных артерий при ГКА не проводили, кроме того, при использовании ПЭТ сложно дифференцировать интра- и экстракраниальные сосуды.

Рекомендация 6. До настоящего времени не выбран приоритетный метод визуализации при подозрении на ГКА с экстракраниальным поражением и преимущественно системными симптомами; могут быть использованы УЗДС, ПЭТ, МРТ и/или КТ-ангиография. Применение ПЭТ может быть особенно актуально у пожилых пациентов с конституциональными симптомами без характерных клинических признаков ГКА для исключения новообразования или инфекции, информативность возрастает при использовании ПЭТ-КТ. УЗДС имеет ограниченную ценность для оценки поражения грудной аорты.

Рекомендация 7. У пациентов с подозрением на АТ приоритетно применение МРТ-ангиографии, что основано на мнении экспертов и современной клинической практике. Дополнительным аргументом в связи с молодым возрастом больных АТ является отсутствие при МРТ лучевой нагрузки.

Рекомендация 8. По мнению экспертов, при подозрении на АТ могут быть использованы ПЭТ, КТ и/или УЗДС. Отмечено, что ПЭТ особенно информативна при наличии неспецифических симптомов АТ для дифференциальной диагностики с другими заболеваниями. УЗДС может быть информативна у больных АТ с поражением сосудов конечностей, но без использования транспищеводного доступа не позволяет визуализировать изменения в грудной аорте.

Рекомендащия 9. Рентген-контрастная ангиография не позволяет охарактеризовать изменения в сосудистой стенке, сложна для выполнения и сопряжена с более высоким риском осложнений по сравнению с другими методами визуализации, в связи с чем не рекомендована для диагностики ГКА или АТ и может быть заменена другими методами исследования. У пациентов с CB крупных сосудов ангиография сохраняет значение для выполнения хирургических вмешательств на сосудах (чрескожная транслюминальная баллонная ангиопластика, стентирование) [56].

Рекомендация 10. Подчеркнуто значение инструментальных методов обследования у пациентов с подозрением на рецидив СВ крупных сосудов. В ряде случаев результаты клинического и лабораторного обследования неубедительны, и визуализация с помощью инструментальных методов может иметь решающее значение для уточнения фазы болезни и определения тактики лечения. Выбор метода определяется клинической ситуацией, доступностью исследований и опытом специалистов. 
У отдельных пациентов различные методы могут иметь взаимодополняющее значение. Вместе с тем при несомненных клинических признаках рецидива или полной клинико-лабораторной ремиссии ценность дополнительного применения инструментального обследования для уточнения активности СВ в настоящее время не установлена и требует изучения.

Рекомендация 11. Мониторинг с использованием инструментального обследования требуется больным ГКА и АТ с развитием стеноза/окклюзии или аневризм крупных сосудов, а также при рецидивах или персистенции сосудистого воспаления; при этом выбор метода визуализации зависит от локализации поражения. Так, воспаление и/или дилатация аорты требуют выполнения МРТ- или КТангиографии, в то время как для визуализации окклюзии/стеноза подмышечной, подключичной артерий можно ограничиться УЗДС. Частоту повторных исследований для оценки динамики стеноза артерий или исключения аневризмы аорты следует определять индивидуально, поскольку в настоящее время недостаточно данных для обоснования определенного протокола мониторинга.

Рекомендация 12. Экспертная группа согласовала необходимость стандартизации процедур исследований с выделением технических требований для получения чувствительных и надежных результатов [57]. Подчеркнута приоритетность специальных программ обучения и международных курсов по инструментальной диагностике СВ крупных сосудов, особенно УЗДС, для успешного внедрения данных рекомендаций EULAR в клиническую практику.

Отсутствие доказательной базы и клинического опыта не позволило сформулировать рекомендации в отношении прогностической ценности инструментальных методов при ГКА и АТ. Рекомендации были дополнены предложениями в отношении техники выполнения и эксплуатации различных методов визуализации, представленными в особом разделе.

Кроме того, эксперты рабочей группы сформулировали дальнейшие перспективные направления, включая: определение золотого стандарта диагностики, в первую очередь ГКА; сравнение диагностической ценности УЗДС, МРТ, КТ и ПЭТ при различных паттернах и локализациях поражения при СВ крупных сосудов; разработку комбинированных методов; изучение значения визуализации для выбора терапии, мониторинга, в том числе аортальных осложнений, и определения прогноза заболевания; сравнение ценности визуализации при участии операторов с низким и высоким опытом в области СB; изучение ценности новых методов, таких как УЗДС с контрастным усилением или ПЭТ с лигандами, специфически нацеленными на иммунные клетки.

Большая часть перспективных направлений касалась различных аспектов УЗДС, поскольку именно этот метод является самым доступным, дешевым, безопасным, а в ряде случаев и наиболее информативным.

Через год после публикации рекомендаций EULAR были представлены результаты ряда исследований, уточняющих возможности УЗДС для выявления воспалительных изменений в сосудах при АТ. Так, в шведском исследовании [58], включавшем 25 пациентов с достоверно установленным АТ, был предложен ультразвуковой индекс АТ (включающий маркерные точки УЗДС сосудов, степень утолщения комплекса «интима - медиа, диаметр сосуда, диаметр просвета сосуда) и выделены пять степеней изменений сосудистой стенки при АТ. Методы УЗДС применяют для визуализации поражения коронарных артерий при АТ [7]. Рекомендации EULAR рассматривали УЗДС с цветовым картированием кровотока, однако за последние годы появились исследования, посвященные усовершенствованным методам ультразвукового исследования с контрастным усилением (КУУЗИ), в определенной степени способные решать ряд задач, поставленных экспертной группой. Так, у пациентов с АТ поглощение радиофармпрепарата при ПЭТ коррелировало с интенсивностью накопления в стенке сосуда контраста при КУУЗИ; кроме того, при КУУЗИ сонных артерий выявляли признаки неоваскуляризации, которые предложено рассматривать в качестве потенциального маркера активности АТ [59-62].

Таким образом, несмотря на значительный прогресс инструментальной диагностики СВ крупных сосудов, требуются дальнейшие исследования, в том числе для уточнения чувствительности различных методов визуализации на ранней стадии СВ или при рецидиве, выделения приоритетных методов в зависимости от клинического варианта и стадии заболевания, а также повышение уровня подготовки ревматологов и специалистов лучевой диагностики и ядерной медицины.

\section{Прозрачность исследования}

Исследование не имело спонсорской поддержки. Авторы несут полную ответственность за предоставление окончательной версии рукописи в печать.

Декларация о финансовых и других взаимоотношениях

Все авторы принимали участие в разработке концепции статьи и в написании рукописи. Окончательная версия рукописи была одобрена всеми авторами. Авторы не получали гонорар за статью.

\section{Л И Т Е Р А У Р A / REFEREN C E S}

1. Jennette JC, Falk RJ, Bacon PA, Basu N, Cid MC, Ferrario F, et al. 2012 revised International Chapel Hill Consensus Conference nomenclature of vasculitides. Arthritis Rheum. 2013;65(1):1-11. doi: 10.1002/art.37715

2. Zarka F, Veillette C, Makhzoum JP. A review of primary vasculitis mimickers based on the Chapel Hill Consensus classification. Int J Rheumatol. 2020;2020:8392542. doi: 10.1155/2020/8392542

3. Peng L, Zhang P, Li J, Liu Z, Lu H, Zhu L, et al. IgG4-related aortitis/periaortitis and periarteritis: A distinct spectrum of IgG4related disease. Arthritis Res Ther. 2020;22(1):103. doi: 10.1186/ s13075-020-02197-w

4. Corominas H, Tsokos M, Quezado M, Tsokos GC. Aneurysm of the ascending aorta in systemic lupus erythematosus: Case

report and review of the literature. Eur J Rheumatol. 2017;4(2):133135. doi: 10.5152/eurjrheum.2017.17066

5. Willett WF 3rd, Kahn MJ, Gerber MA. Lupus aortitis: A case report and review of the literature. $J$ La State Med Soc. 1996;148(2):55-59.

6. Kaneko S, Yamashita H, Sugimori Y, Takahashi Y, Kaneko H, Kano T, et al. Rheumatoid arthritis-associated aortitis: A case report and literature review. Springerplus. 2014;3:509. doi: 10.1186/2193-1801-3-509

7. Shimizu T, Sato A, Sakamoto K, Seino Y, Kijima M, Matsumoto T, et al. Intravascular ultrasound imaging of isolated and non aorto-ostial coronary Takayasu arteritis: A case report. BMC Cardiovasc Disord. 2020;20(1):260. doi: 10.1186/s12872-020-01541-X 
8. Batra K, Chamarthy M, Chate RC, Jordan K, Kay FU. Pulmonary vasculitis: diagnosis and endovascular therapy. Cardiovasc Diagn Ther. 2018;8(3):297-315. doi: 10.21037/ cdt.2017.12.06

9. Sharma BK, Jain S, Suri S, Numano F. Diagnostic criteria for Takayasu arteritis. Int J Cardiol. 1996;54(Suppl):S141-S147.

10. Hata A, Noda M, Moriwaki R, Numano F. Angiographic findings of Takayasu arteritis: New classification. Int J Cardiol. 1996;54(Suppl):S155-S163.

11. Moriwaki R, Noda M, Yajima M, Sharma BK, Numano F. Clinical manifestations of Takayasu arteritis in India and Japan new classification of angiographic findings. Angiology. 1997;48(5):369-379. doi: 10.1177/000331979704800501

12. Покровский АВ, Зотиков АЕ, Юдин ВИ. Неспецифический аортоартериит (болезнь Такаясу). М.:ИРСИСъ;2002. [Pokrovskii AV, Zotikov AE, Yudin VI. Nonspecific aortoarteritis (Takayasu's disease). Moscow:IRSIS;2002 (In Russ.)].

13. Hong S, Bae SH, Ahn SM, Lim DH, Kim YG, Lee CK, et al. Outcome of Takayasu arteritis with inactive disease at diagnosis: The extent of vascular involvement as a predictor of activation. J Rheumatol. 2015;42(3):489-494. doi: 10.3899/jrheum.140981

14. Blockmans D, Coudyzer W, Vanderschueren S, Stroobants S, Loeckx D, Heye S, et al. Relationship between fluorodeoxyglucose uptake in the large vessels and late aortic diameter in giant cell arteritis. Rheumatology (Oxford). 2008;47(8):1179-1184. doi: 10.1093/rheumatology/ken 119

15. Robson JC, Kiran A, Maskell J, Hutchings A, Arden N, Dasgupta B, et al. The relative risk of aortic aneurysm in patients with giant cell arteritis compared with the general population of the UK. Ann Rheum Dis. 2015;74(1):129-135. doi: 10.1136/annrheumdis-2013-204113

16. Dejaco C, Ramiro S, Duftner C, Besson FL, Bley TA, Blockmans D, et al. EULAR recommendations for the use of imaging in large vessel vasculitis in clinical practice. Ann Rheum Dis. 2018;77(5):636-643. doi: 10.1136/annrheumdis-2017-212649

17. Hellmich B, Agueda A, Monti S, Buttgereit F, de Boysson H, Brouwer E, et al. 2018 update of the EULAR recommendations for the management of large vessel vasculitis. Ann Rheum Dis. 2020;79(1):19-30. doi: 10.1136/annrheumdis-2019-215672

18. Duftner C, Dejaco C, Sepriano A, Falzon L, Schmidt WA, Ramiro S. Imaging in diagnosis, outcome prediction and monitoring of large vessel vasculitis: A systematic literature review and meta-analysis informing the EULAR recommendations. RMD Open. 2018;4(1):e00612. doi: 10.1136/rmdopen-2017-000612

19. Aschwanden M, Daikeler T, Kesten F, Baldi T, Benz D, Tyndall A, et al. Temporal artery compression sign - A novel ultrasound finding for the diagnosis of giant cell arteritis. Ultraschall Med. 2013;34(1):47-50. doi: 10.1055/s-0032-1312821

20. Aschwanden M, Imfeld S, Staub D, Baldi T, Walker UA, Berger CT, et al. The ultrasound compression sign to diagnose temporal giant cell arteritis shows an excellent interobserver agreement. Clin Exp Rheumatol. 2015;33(2 Suppl 89):S113-S115.

21. Salvarani C, Cantini F, Boiardi L, Hunder G. Laboratory investigations useful in giant cell arteritis and Takayasuэs arteritis. Clin Exp Rheum. 2003;21(6 Suppl 32):23-28.

22. Kerr GS, Hallahan CW, Giordano J, Leavitt RY, Fauci AS, Rottem M, et al. Takayasu arteritis. Ann Intern Med. 1994;120(11):919-929. doi: 10.7326/0003-4819-120-11-19940601000004

23. Xenitidis T, Horger M, Zeh G, Kanz L, Henes JC. Sustained inflammation of the aortic wall despite tocilizumab treatment in two cases of Takayasu arteritis. Rheumatology (Oxford). 2013;52(9):1729-1731. doi: 10.1093/rheumatology/ket107

24. Spira D, Xenitidis T, Henes J, Horger M. MRI parametric monitoring of biological therapies in primary large vessel vasculitides: A pilot study. Br J Radiol. 2016;89(1058):20150892. doi: 10.1259/ bjr.20150892

25. García-Martínez A, Arguis P, Prieto-González S, EspígolFrigolé G, Alba MA, Butjosa M, et al. Prospective long term follow-up of a cohort of patients with giant cell arteritis screened for aortic structural damage (aneurysm or dilatation). Ann Rheum Dis. 2014;73(10):1826-1832. doi: 10.1136/annrheumdis-2013-203322

26. Mavrogeni S, Dimitroulas T, Chatziioannou SN, Kitas G. The role of multimodality imaging in the evaluation of Takayasu arteritis. Semin Arthritis Rheum. 2013;42(4):401-412. doi: 10.1016/ j.semarthrit.2012.07.005

27. Schmidt WA. Imaging in vasculitis. Best Pract Res Clin Rheumatol. 2013;27:107-118. doi: 10.1016/j.berh.2013.01.001

28. Muratore F, Pipitone N, Salvarani C, Schmidt WA. Imaging of vasculitis: State of the art. Best Pract Res Clin Rheumatol. 2016;30(4):688-706. doi: 10.1016/j.berh.2016.09.010

29. Germanò G, Monti S, Ponte C, Possemato N, Caporali R, Salvarani $\mathrm{C}$, et al. The role of ultrasound in the diagnosis and follow-up of large-vessel vasculitis: An update. Clin Exp Rheumatol. 2017;35(Suppl 103(1)):194-198.

30. Schmidt WA, Kraft HE, Vorpahl K, Völker L, Gromnica-Ihle EJ. Color duplex ultrasonography in the diagnosis of temporal arteritis. N Engl J Med. 1997;337(19):1336-1342. doi: 10.1056/ NEJM199711063371902

31. Karahaliou M, Vaiopoulos G, Papaspyrou S, Kanakis MA, Revenas K, Sfikakis PP. Colour duplex sonography of temporal arteries before decision for biopsy: a prospective study in 55 patients with suspected giant cell arteritis. Arthritis Res Ther. 2006;8(4):R116. doi: 10.1186/ar2003

32. De Miguel E, Roxo A, Castillo C, Peiteado D, Villalba A, MartínMola E. The utility and sensitivity of colour Doppler ultrasound in monitoring changes in giant cell arteritis. Clin Exp Rheumatol. 2012;30(1 Suppl 70):S34-S38.

33. Habib HM, Essa AA, Hassan AA. Color duplex ultrasonography of temporal arteries: Role in diagnosis and follow-up of suspected cases of temporal arteritis. Clin Rheumatol. 2012;31:231-237. doi: 10.1007/s10067-011-1808-0

34. Pérez López J, Solans Laqué R, Bosch Gil JA, Molina Cateriano C, Huguet Redecilla P, Vilardell Tarrés M. Colour-duplex ultrasonography of the temporal and ophthalmic arteries in the diagnosis and follow-up of giant cell arteritis. Clin Exp Rheumatol. 2009;27(1 Suppl 52):S77-S82.

35. Schmidt WA, Moll A, Seifert A, Schicke B, Gromnica-Ihle E, Krause A. Prognosis of large-vessel giant cell arteritis. Rheumatology (Oxford). 2008;47(9):1406-1408. doi: 10.1093/rheumatology/ken 258

36. Pipitone N, Versari A, Hunder GG, Salvarani C. Role of imaging in the diagnosis of large and medium-sized vessel vasculitis. Rheum Dis Clin North Am. 2013;39:593-608. doi: 10.1016/j.rdc.2013.02.002

37. Prieto-González S, Arguis P, Cid MC. Imaging in systemic vasculitis. Curr Opin Rheumatol. 2015;27:53-62. doi: 10.1097/ BOR.0000000000000130

38. Blockmans D, de Ceuninck L, Vanderschueren S, Knockaert D, Mortelmans L, Bobbaers H. Repetitive 18F-fluorodeoxyglucose positron emission tomography in giant cell arteritis: A prospective study of 35 patients. Arthritis Rheum. 2006;55(1):131-137. doi: 10.1002/art.21699

39. Lariviere D, Benali K, Coustet B, Pasi N, Hyafil F, Klein I, et al. Positron emission tomography and computed tomography angiography for the diagnosis of giant cell arteritis: A real-life prospective study. Medicine (Baltimore). 2016;95(30):e4146. doi: 10.1097/ MD.0000000000004146

40. Blockmans D, Stroobants S, Maes A, Mortelmans L. Positron emission tomography in giant cell arteritis and polymyalgia rheumatica: Evidence for inflammation of the aortic arch. Am J Med. 2000;108(3):246-249. doi: 10.1016/s0002-9343(99)00424-6

41. Puppo C, Massollo M, Paparo F, Camellino D, Piccardo A, Shoushtari Zadeh Naseri M, et al. Giant cell arteritis: A systematic review of the qualitative and semiquantitative methods to assess vasculitis with $18 \mathrm{~F}$-fluorodeoxyglucose positron emission tomography. Biomed Res Int. 2014;2014:574248. doi: 10.1155/2014/574248

42. Bucci M, Aparici CM, Hawkins R, Bacharach S, Schrek C, Cheng $\mathrm{S}$, et al. Validation of FDG uptake in the arterial wall as an imaging biomarker of atherosclerotic plaques with $18 \mathrm{~F}$-fluorodeoxyglucose positron emission tomography-computed tomo- 
graphy (FDG-PET/CT). J Neuroimaging. 2014;24(2):117-123. doi: 10.1111/j.1552-6569.2012.00740.x

43. Direskeneli H. Clinical assessment in Takayasu's arteritis: Major challenges and controversies. Clin Exp Rheumatol. 2017;35:189-193.

44. Oura K, Yamaguchi Oura M, Itabashi R, Maeda T. Vascular Imaging Techniques to Diagnose and Monitor Patients with Takayasu Arteritis: A Review of the Literature. Diagnostics (Basel). 2021;11(11):1993. doi: 10.3390/diagnostics11111993.

45. Park JH, Chung JW, Lee KW, Park YB, Han MC. CT angiography of Takayasu arteritis: Comparison with conventional angiography. J Vasc Interv Radiol. 1997;8(3):393-400. doi: 10.1016/s10510443(97)70579-7

46. Paul JF, Fiessinger JN, Sapoval M, Hernigou A, Mousseaux E, Emmerich J, et al. Follow-up electron beam CT for the management of early phase Takayasu arteritis. J Comput Assist Tomogr. 2001;25(6):924-931. doi: 10.1097/00004728-200111000-00015

47. Yamada I, Nakagawa T, Himeno Y, Numano F, Shibuya $H$. Takayasu arteritis: Evaluation of the thoracic aorta with CT angiography. Radiology. 1998;209(1):103-109. doi: 10.1148/ radiology.209.1.9769819

48. Li D, Lin J, Yan F. Detecting disease extent and activity of Takayasu arteritis using whole-body magnetic resonance angiography and vessel wall imaging as a 1-stop solution. J Comput Assist Tomogr. 2011;35:468-474. doi: 10.1148/radiology.209.1.9769819

49. Yamada I, Nakagawa T, Himeno Y, Kobayashi Y, Numano F, Shibuya H. Takayasu arteritis: Diagnosis with breath-hold contrast-enhanced three-dimensional MR angiography. J Magn Reson Imaging. 2000;11(5):481-487. doi: 10.1002/(sici) 1522-2586(200005) 11:5<481::aid-jmri3>3.0.co;2-4

50. Salvarani C, Silingardi M, Ghirarduzzi A, Lo Scocco G, Macchioni P, Bajocchi G, et al. Is duplex ultrasonography useful for the diagnosis of giant-cell arteritis? Ann Intern Med. 2002;137(4):232-238. doi: 10.7326/0003-4819-137-4-20020820000006

51. Bley TA, Reinhard M, Hauenstein C, Markl M, Warnatz K, Hetzel A, et al. Comparison of duplex sonography and high-resolution magnetic resonance imaging in the diagnosis of giant cell (temporal) arteritis. Arthritis Rheum. 2008;58(8):2574-2578. doi: 10.1002/art.23699

52. Zhang B, Liang L, Chen W, Liang C, Zhang S. An updated study to determine association between gadolinium-based contrast agents and nephrogenic systemic fibrosis. PLoS One. 2015;10(6):e0129720. doi: 10.1371/journal.pone. 0129720

Бекетова T.B. ORCID: https://orcid.org/0000-0003-2641-9785

Зеленов B.A. ORCID: https://orcid.org/0000-0002-7064-5301

Зотиков A.E. ORCID: https://orcid.org/0000-0002-1688-7756
53. Kanda T, Nakai Y, Hagiwara A, Oba H, Toyoda K, Furui S. Distribution and chemical forms of gadolinium in the brain: A review. Br J Radiol. 2017;90(1079):20170115. doi: 10.1259/ bjr. 20170115

54. OCEBM Levels of Evidence Working Group. The Oxford 2011 levels of evidence. 2011. URL: http://www.cebm.net/index.aspx$? \mathrm{o}=5653$.

55. Diamantopoulos AP, Haugeberg G, Hetland H, Soldal DM, Bie R, Myklebust G. Diagnostic value of color Doppler ultrasonography of temporal arteries and large vessels in giant cell arteritis: A consecutive case series. Arthritis Care Res (Hoboken). 2014;66(1):113-119. doi: 10.1002/acr.22178

56. Gülcü A, Gezer NS, Akar S, Akkoç N, Önen F, Göktay AY. Longterm follow-up of endovascular repair in the management of arterial stenosis caused by Takayasu's arteritis. Ann Vasc Surg. 2017;42:93-100. doi: 10.1016/j.avsg.2016.10.066

57. De Miguel E, Castillo C, Rodríguez A, De Agustín JJ; Working Group Ultrasound Giant Cell Arteritis. Learning and reliability of colour Doppler ultrasound in giant cell arteritis. Clin Exp Rheumatol. 2009;27(1 Suppl 52):S53-S58.

58. Svensson C, Eriksson P, Zachrisson H. Vascular ultrasound for monitoring of inflammatory activity in Takayasu arteritis. Clin Physiol Funct Imaging. 2020;40(1):37-45. doi: 10.1111/ cpf.12601

59. Magnoni M, Dagna L, Coli S, Cianflone D, Sabbadini MG, Maseri A. Assessment of Takayasu arteritis activity by carotid contrast-enhanced ultrasound. Circ Cardiovasc Imaging. 2011;4(2):e1e2. doi: 10.1161/CIRCIMAGING.110.960906

60. Giordana P, Baqué-Juston MC, Jeandel PY, Mondot L, Hirlemann J, Padovani B, et al. Contrast-enhanced ultrasound of carotid artery wall in Takayasu disease: First evidence of application in diagnosis and monitoring of response to treatment. Circulation. 2011;124(2):245-247. doi: 10.1161/ CIRCULATIONAHA.110.006668

61. Possemato N, Macchioni P, Germanò G, Pipitone N, Versari A, Salvarani C. Clinical images: PET-CT and contrast-enhanced ultrasound in Takayasu's arteritis. Rheumatology (Oxford). 2014;53(3):447. doi: 10.1093/rheumatology/ket332

62. Schinkel AF, van den Oord SC, van der Steen AF, van Laar JA, Sijbrands EJ. Utility of contrast-enhanced ultrasound for the assessment of the carotid artery wall in patients with Takayasu or giant cell arteritis. Eur Heart J Cardiovasc Imaging. 2014;15:541546. doi: $10.1093 /$ ehjci/jet243 